\title{
Childhood tuberculosis in the European Union/ European Economic Area, 2000 to 2009
}

A Sandgren ${ }^{1}$, V Hollo ${ }^{1}$, C Quinten², D Manissero (davide.manissero@ecdc.europa.eu)1

1. Tuberculosis Disease Specific Programme, European Centre for Disease Prevention and Control (ECDC), Stockholm, Sweden

2. Surveillance Unit, European Centre for Disease Prevention and Control (ECDC), Stockholm, Sweden

Sandgren A, Hollo V, Quinten C, Manissero D. Childhood tuberculosis in the European Union/European Economic Area, 2000 to 2009. Euro Surveill.

2011;16(12):pii=19825. Available online: http://www.eurosurveillance.org/ViewArticle.aspx?Articleld=19825

Article published on 24 March 2011

Childhood tuberculosis (TB) has been neglected for decades as a key component of TB control. However, ensuring proper monitoring of childhood TB has recently been given renewed emphasis. A descriptive analysis of surveillance data was performed to assess burden and trends of paediatric TB in the European Union/European Economic Area (EU/EEA) between 2000 and 2009. From 2000 to 2009, 39,695 notified paediatric (defined as 0-14 years of age) TB cases were reported by the 27 EU countries plus Norway, Iceland and Liechtenstein. These paediatric cases accounted for $4.3 \%$ of all notified cases. However, across the EU/EEA Member States, paediatric case notification rates ranged from 29.6 per 100,000 to 0.3 per 100,000 for the latest reporting year, 2009. Overall, though, these rates dropped from 5.5 per 100,000 in 2000 to 4.2 per 100,000 in 2009. The EU/EEA average annual percent changes (AAPC) in paediatric notification rates decreased between 2000 and 2004 by $1.3 \%$ and between 2005 and 2009 by $2.4 \%$, with an overall decrease between 2000 and 2009 of $2.8 \%$. Of all paediatric cases reported from 2000 to 2009 , only $16.9 \%$ were culture-confirmed, amongst which the overall treatment success was $80.5 \%$ for all culture-confirmed pulmonary paediatric TB cases. Childhood TB in the EU/EEA remains a public health issue. Due attention should be paid to assessing paediatric trends as they could provide an insight in recent transmission. Whilst the primary aim of further reducing TB rates among children is paramount, better rates of appropriate diagnosis should also be achieved, along with a further improvement of therapeutic success rates.

\section{Introduction}

Childhood tuberculosis (TB) has long been an overlooked area within global TB control [1]. However, of the estimated 9 million cases of TB occurring annually in the world, approximately 1 million occur in children under the age of 15 years. Seventy-five per cent of the global childhood TB burden occurs in the 22 high-burden countries, where the proportion attributable to children ranges from $15 \%$ to $20 \%$ of all cases. In contrast, in low-burden countries, this proportion is reported to be approximately $5 \%$ [1].
It is, however, interesting to note that given the recent changes in the TB epidemic in low-incidence countries, with a resurgence of cases and a failure in further TB decline, we now see a renewed emphasis on ensuring correct monitoring of childhood TB [2-5]. In particular, the notion that childhood TB represents a sentinel event of ongoing transmission, and that children rapidly progress to active disease and represent a potential pool for disease in their adult life, further underlines the importance of analysing the epidemiology of childhood TB.

Despite previous attempts to provide an overview of the European situation [6], an in-depth analysis of childhood TB in the Member States of the European Union (EU) and European Economic Area (EEA) has not previously been undertaken. Epidemiological analysis of EU data over the past years [7-8] has highlighted the need to further describe the trends in paediatric TB in the EU/EEA Member States. The analysis presented here aims to provide a descriptive and analytic overview of the trends in childhood TB notifications, diagnosis and treatment outcome between 2000 and 2009.

\section{Methods}

Data source and collection

A descriptive analysis of surveillance data was performed to assess burden and trends of paediatric TB in EU/EEA countries between January 2000 and December 2009. Data for the years 2007 to 2009 were extracted from The European Surveillance System (TESSy), and for years 2000 to 2006 from the historical databases of the former EURO-TB network, the Individual Database of Tuberculosis (EITUD) and the aggregated database (TABORIG), held at the European Centre for Disease Prevention and Control (ECDC). Data from the $30 \mathrm{EU}$ and EEA countries reporting to the ECDC were analysed. Not all countries have reported data for the whole period from 2000 to 2009 , therefore we indicate, where appropriate, the number of countries the presented data are based on.

For the purposes of this study, country-specific data for cases under the age of 15 years were extracted for 
the years of analysis, for both new and retreatment pulmonary and extra-pulmonary TB cases. Since the reporting year 2002, treatment outcome data have been collected for all individual cases. The cases eligible for analysis (cohorts) included all TB cases notified in the calendar year of interest, after exclusion of cases with final diagnosis other than TB. For comparison between paediatric and overall trends, we used the trends from the latest reported national five-year notification included in the TB surveillance report for 2009 [9].

\section{Data inclusions and surveillance definitions}

All TB cases, confirmed, probable or possible [7], notified at country level for the year of interest are included in the dataset uploaded to TESSy and were included in the study if they were under 15 years-old. Cases eligible for treatment but who never started treatment were also included, as well as cases diagnosed post mortem. Pulmonary TB was defined as TB affecting the lung parenchyma, the tracheobronchial tree or the larynx. Extrapulmonary TB was defined as TB with nonpulmonary presentations, and includes pleural and mediastinal/hilar forms. All definitions and categories of the data analysed are consistent with those published in the Tuberculosis surveillance in Europe 2008 report [7].

For the purpose of disaggregated data analysis, countries were grouped in high- and low-incidence TB countries, using the thresholds previously proposed by the Wolfeheze working group [10] and recently adopted in the EU monitoring framework [5]. Thus, low-incidence countries were defined as those with less than 20 cases per 100,000 population, of which there were 23 countries, and high-incidence countries as those with 20 or more cases per 100,000 population, which comprised seven countries: Bulgaria, Estonia, Latvia, Lithuania, Poland, Portugal and Romania.

\section{Data quality}

The data uploaded to TESSy went through automated checks for completeness and accuracy. Until 2007, only 27 out of the $30 \mathrm{EU} / \mathrm{EEA}$ current Member States were able to report case-based data. Aggregated data for paediatric cases allowed only analysis of the origin of cases and site of the disease.

\section{Analysis}

STATA 11 (StataCorp LP, College Station, Texas, USA) and Microsoft Excel 2007 were used for data analyses. The collected data from 2000 to 2009 were collated and tabulated in an aggregated fashion. Population size was obtained from the EUROSTAT database for the period 2000 to 2009.

Childhood TB rates were calculated as age-specific notification rates for the population aged o to 14 years. The trends in notification rates were expressed as the average annual percentage change (AAPC) $[7,8]$. The AAPC was calculated for the whole period from 2000 to
2009, and in some cases from 2000 to 2004 and from 2005 to 2009 , to allow comparisons with previously published trends in the EU $[7,8]$. The relation between national AAPC of paediatric cases versus that of all TB cases was analysed using the Pearson correlation $\left(R^{2}\right)$. Countries with less than 10 cases per year for each year between 2005 and 2009 were excluded from the analyses, to avoid introducing bias from countries with low absolute numbers of cases, where the AAPC would be strongly affected by small absolute changes. Separate analyses were done for low-incidence countries and high-incidence countries, to investigate possible differences in trends between these two distinct epidemiological settings.

Linear regression models were applied to investigate statistically significant differences in time trends of notification rates between $0-4$ year-olds and 5-14 year-olds, and between high- and low- incidence countries for the age groups of under one year-olds, 1-4 year-olds, as well as all paediatric cases. The Fisher's z-transformation was used to compare correlation coefficients. Fisher's exact test or chi-square tests were used to compare proportions and notification rates between groups. A p 0.05 was considered as statistically significant.

\section{Results}

Paediatric tuberculosis notification and trends During the period 2000 to 2009 , 39,695 notified paediatric TB cases were reported by 27 EU countries plus Norway, Iceland and Liechtenstein: these accounted for $4.3 \%$ of the total TB burden in the EU/EEA. The notification rates of paediatric cases ranged from 29.6 per 100,000 to 0.3 per 100,000 across the EU/EEA Member States for the latest reporting year 2009. During the ten years, the EU/EEA overall paediatric notification rates dropped from 5.5 per 100,000 in 2000 to 4.2 per 100,000 in 2009. The EU/EEA AAPC in paediatric notification rates decreased between 2000 and 2004 by $1.3 \%$ and between 2005 and 2009 by $2.4 \%$, with an overall decrease between 2000 and 2009 of $2.8 \%$ (five-year interval trends will allow a better comparison with previously published data on trends in EU/EEA [8]). However, 13 countries had an increase in notification rates between 2000 and 2004, 15 countries had an increase between 2005 and 2009, and seven had a stable increase for both of these periods.

When excluding the five countries that did not report data for all years, 11 countries, including four high-incidence countries, had a decrease in AAPC in paediatric notification rates for the period from 2000 to 2009. Conversely, 14 countries had an increase in AAPC in notification rates, three of them high-incidence countries (Figure 1).

Correlation analysis between AAPC (2005-2009) for paediatric and all cases (data from $[7,8]$ ), excluding countries with less than 10 cases per year during that period, showed a poor overall correlation $\left(R^{2}=0.32\right.$, 
Average annual percentage change of childhood tuberculosis notification rates for EU/EEA countries, 2000-2009 ( $\mathrm{n}=25$ )

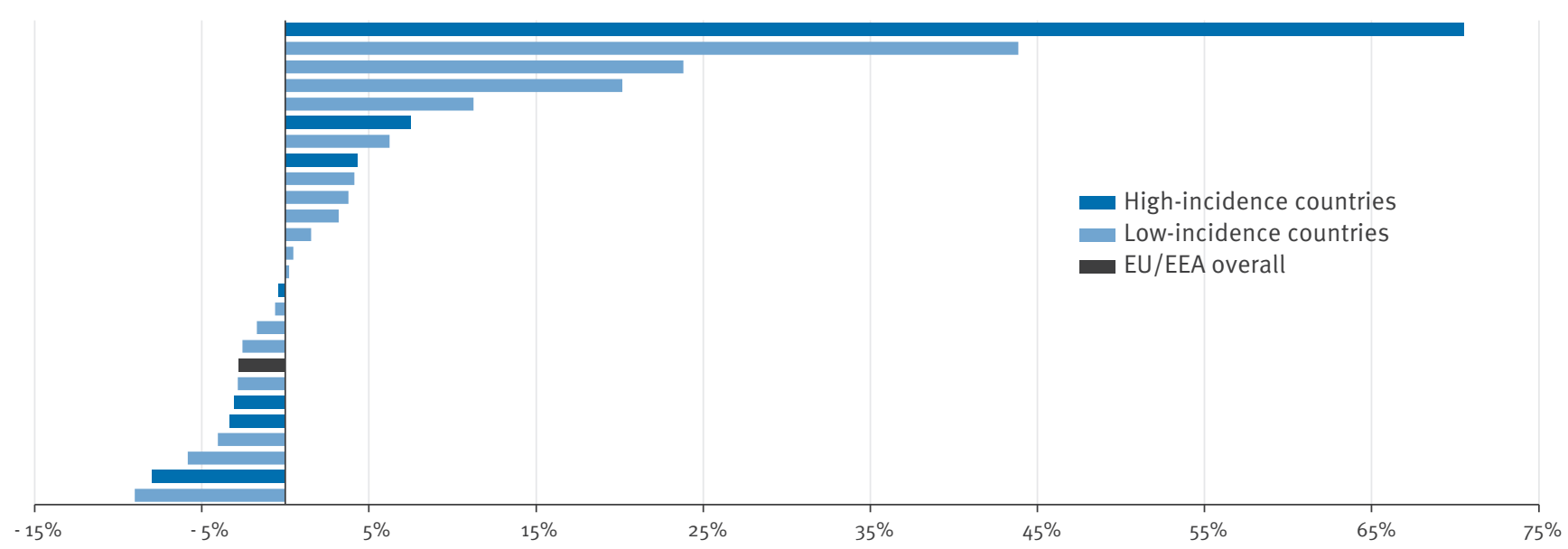

EU/EEA: European Union/European Economic Area.

\section{FIGURE 2}

Correlation between average annual percentage change of all cases and of paediatric cases in (A) low-incidence countries $(\mathrm{n}=14)$ and $(\mathrm{B})$ high-incidence countries $(\mathrm{n}=6)$, EU/EEA, 2005-2009

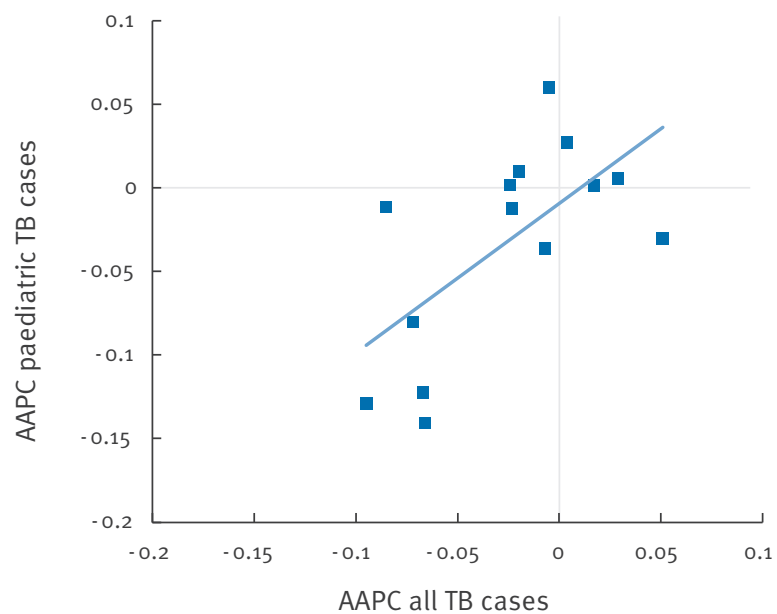

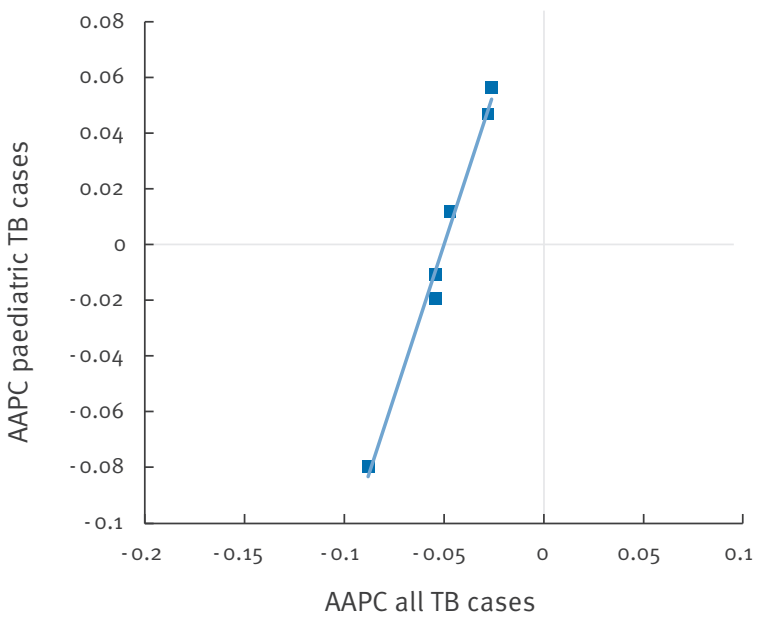

AAPC: average annual percentage change; EU/EEA: European Union/European Economic Area; TB: tuberculosis.

\section{FIGURE 3}

Trends of tuberculosis notification rate in all age groups, EU/EEA, 2000-2009

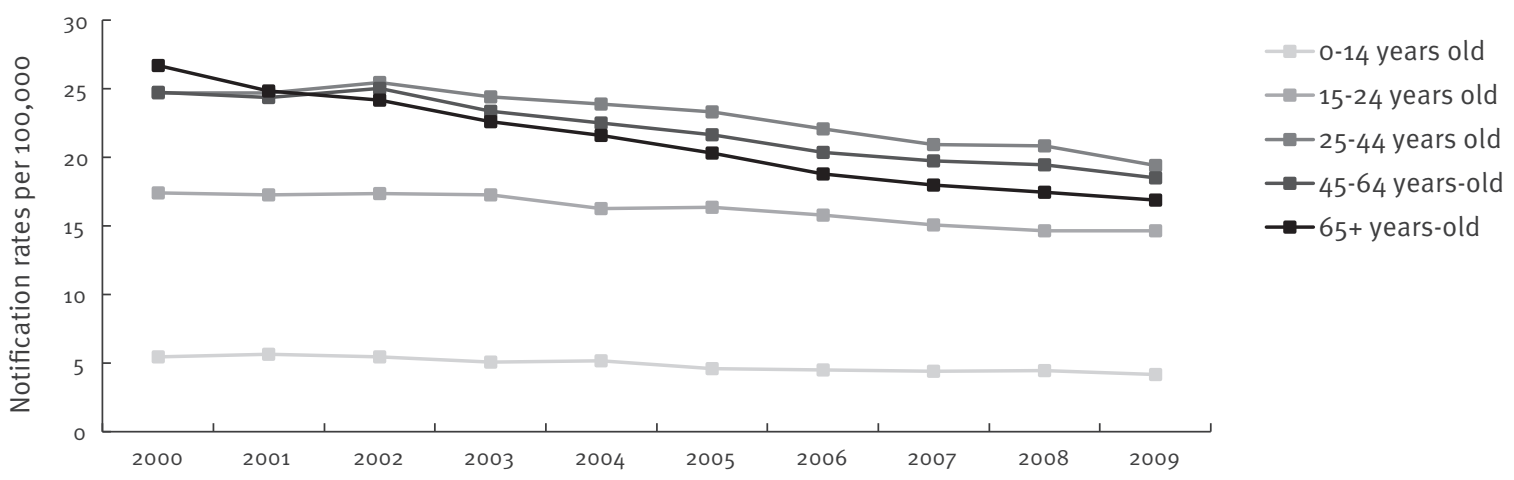

EU/EEA: European Union/European Economic Area. 
$\mathrm{n}=20$ ). Closer investigation however, revealed that this correlation was significantly weaker for low-incidence countries $\left(R^{2}=0.42, n=14\right)$ (Figure $\left.2 A\right)$ than for highincidence countries $\left(R^{2}=0.99, n=6\right)$ (Fisher $Z=-3.17$, $\mathrm{p}=0.0015$ ) (Figure $2 \mathrm{~B}$ ).

Although the notification rates for children were lower than those for other age groups (Figure 3), very young children ( $<5$ years-old), who represented $48 \%$ of all paediatric cases, stood out as having significantly higher notification rates than the 5-14 year-olds (ranges: $5.42-9.21$ per 100,000 in $<5$ year-olds and 3.03-3.91 per 100,000 in 5-14 year-olds; p<0.001).

Although high-incidence countries contribute most paediatric cases, we observed decreases in the AAPC

\section{FIGURE 4}

Paediatric age-specific tuberculosis trends in (A) high-incidence and (B) low-incidence countries, EU/EEA, 2000-2009
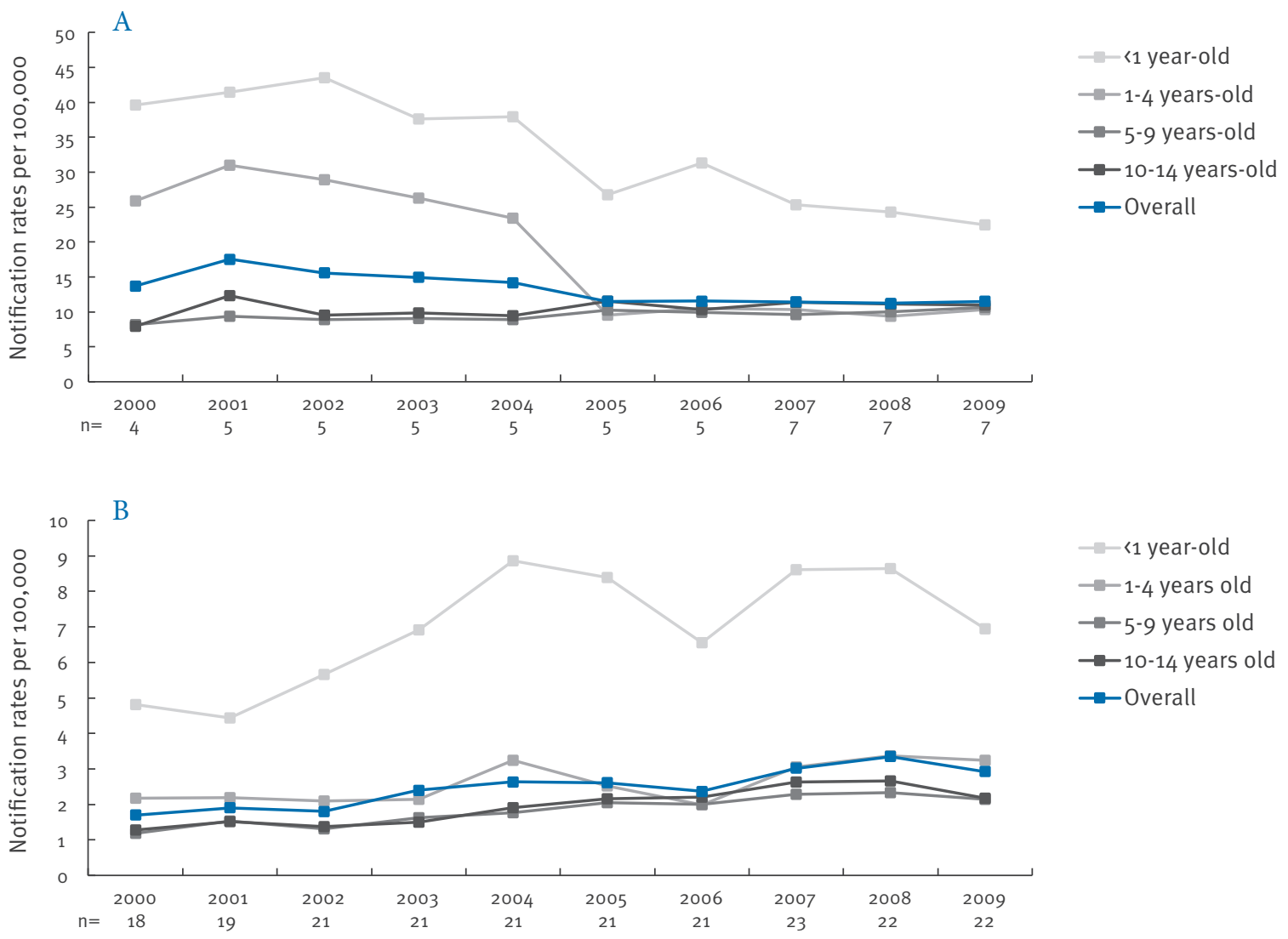

EU/EEA: European Union/European Economic Area.

$\mathrm{n}$ : number of countries reporting data for the particular year.

\section{FIGURE 5}

Proportion of tuberculosis cases by age group and origin of total paediatric cases in low-incidence countries, EU/EEA, 2000-2009
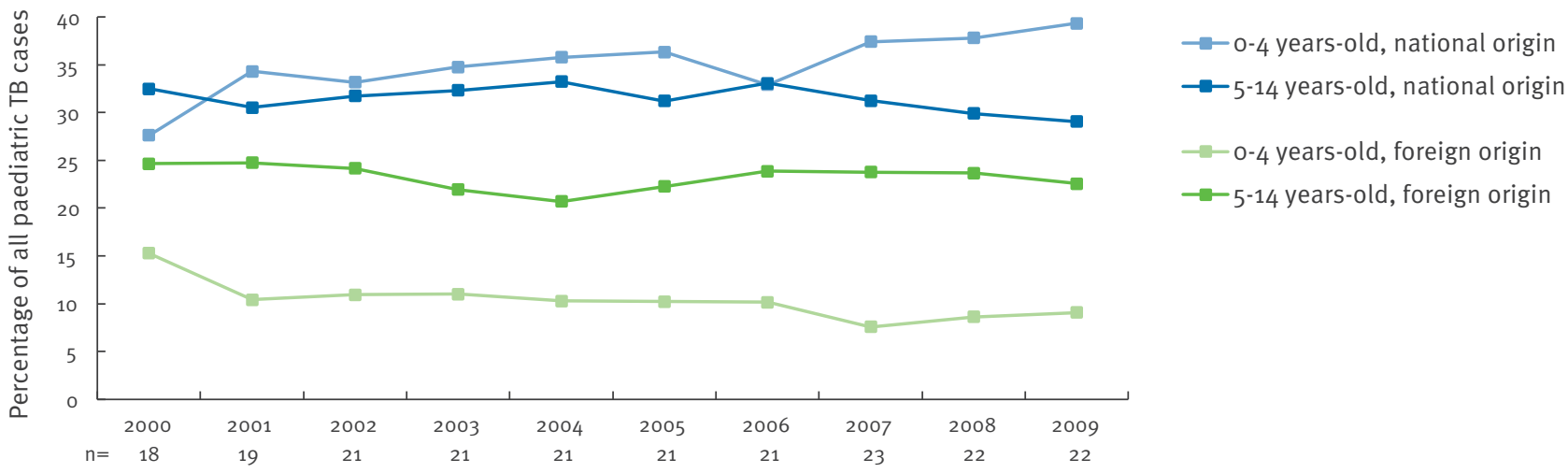

EU/EEA: European Union/European Economic Area.

$\mathrm{n}$ : number of countries reporting data for the particular year. 


\section{FIGURE 6}

Culture-positive paediatric tuberculosis cases and their proportion with respect to the total paediatric cases, EU/EEA, 2000-2009

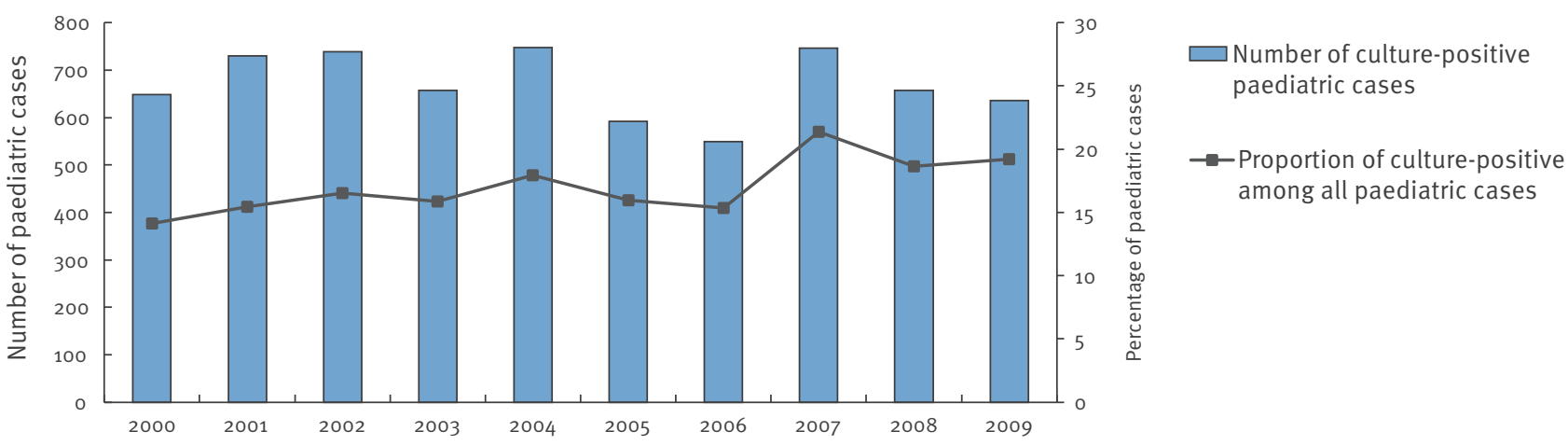

EU/EEA: European Union/European Economic Area; FO: foreign origin; NP: national origin; TB: tuberculosis.

\section{FIGURE 7}

Anti-tuberculosis drug resistance among paediatric cases, EU/EAA, 2000-2009

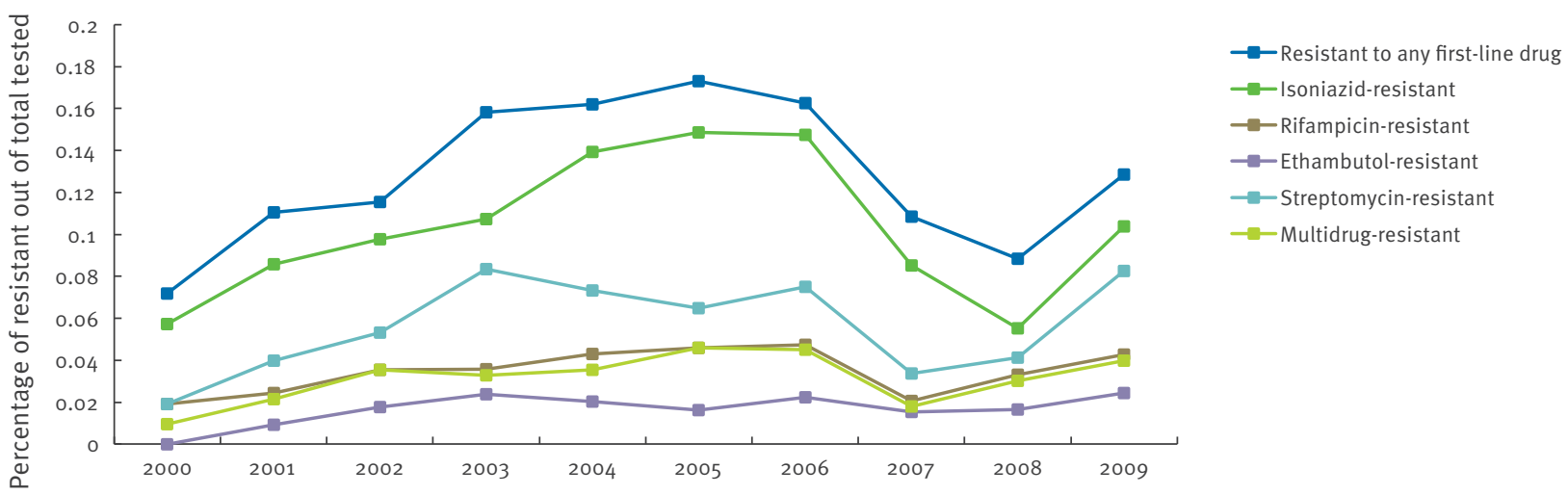

EU/EEA: European Union/European Economic Area.

\section{FIGURE 8}

Treatment outcome for new pulmonary culture-confirmed paediatric tuberculosis cases in high- and low-incidence EU/EEA countries, 2002-2008
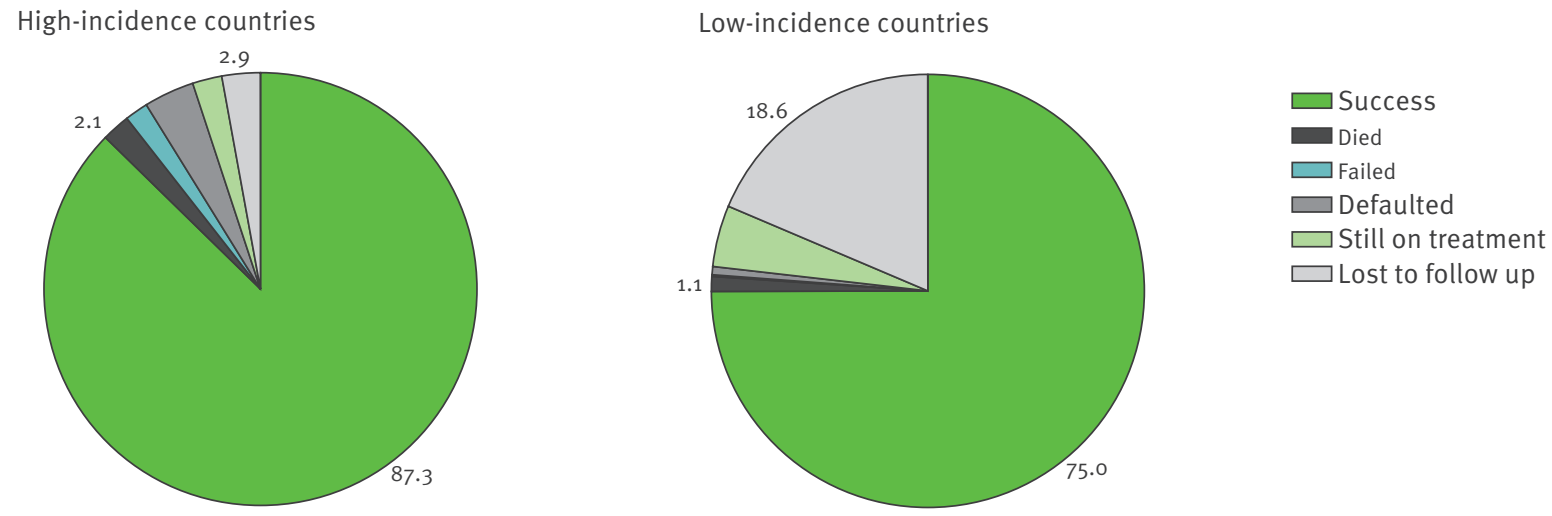

EU/EEA: European Union/European Economic Area. 
of paediatric notification rates between 2000 and 2009: by $5.1 \%$ for the under 1 year-olds and $6.2 \%$ for the 1-4 year-olds. In contrast, in low-incidence countries, there were increases in the AAPC in paediatric notification rates between 2000 and 2009 , by $6.1 \%$ for the under 1 year-olds and $7.4 \%$ for the $1-4$ year-olds (Figure 4). The trends in notified cases for the under 1 year-olds, the 1-4 year-olds and all paediatric cases differed significantly between high- and low-incidence in the period from 2000 to 2009 ( $p<0.001$ for all three comparisons).

\section{Origin of cases}

From 2000 to $2009,15.3 \%$ of the 39,695 paediatric cases were registered as of foreign origin overall in Europe. Most of these foreign cases were in low-incidence countries, where $29.2 \%$ of the paediatric cases were of foreign origin, as opposed to $0.6 \%$ in highincidence countries. The proportion of unknown origin decreased during the study period, but $5.2 \%$ were still of unknown origin in low-incidence countries in 2009. When these cases of unknown origin were excluded, the relative proportions of foreign-born and nationalborn cases appeared stable in low-incidence countries for all paediatric age-groups during the period (Figure 5).

\section{Classification and bacterial \\ confirmation of cases}

From 2000 to $2009,76.7 \%$ of the reported cases had been previously untreated; this proportion was higher $(89.9 \%)$ in the last three years, 2007 to 2009 . For countries that reported on previous treatment, the proportion of previously treated remained stable during the study period (range: $1.1 \%-2.1 \%$ ). In contrast, the proportion of unknown status regarding previous treatment decreased.

Pulmonary TB accounted for $53.4 \%$ of all paediatric TB cases, but only $14.1 \%$ of these were sputum smearpositive. Extra-pulmonary TB accounted for $27.8 \%$ of all paediatric cases, of which $64.4 \%$ had intra-thoracic or pleural TB. Unknown site of disease was frequent among children overall: $18.4 \%$, with higher proportions for those of foreign compared with national origin. Nevertheless, the proportion with unknown site of disease significantly dropped during the study period (from $33.7 \%$ in 2000 to $1.1 \%$ in 2009 , pro.001).

Of the paediatric cases reported between 2000 and $2009(n=39,695)$, only $42.3 \%$ of all paediatric cases were tested by culture; of these $39.9 \%$ were culturepositive. During that period, the AAPC in proportion of culture-tested cases increased by $5.9 \%$. Thus, case confirmation after culture was $16.9 \%$ among all paediatric cases, although its range varied widely across countries. However, the AAPC in the proportion of culture-confirmed cases increased by $4.5 \%$ during the study period. Nevertheless the proportion of cultureconfirmed cases in 2009 was still only $19.2 \%$, compared to $14.1 \%$ in 2000 (Figure 6).

\section{Drug-resistant TB}

The proportion of notified cases for whom drug susceptibility testing (DST) was performed increased from $4.6 \%$ of 4,589 cases in 2000 to $9.9 \%$ of 3,308 cases in 2009 ( $p<0.001$ ), but levelled off after 2006. The same trend was seen for the proportion of culture-positive cases with a DST, which increased from $32.3 \%$ of 648 in 2000 to $51.4 \%$ of 636 in 2009 (p<0.001). Until 2006, there was a steady increase in resistance rates to firstline drugs and isoniazid (Figure 7). However, after 2006, this trend was interrupted, only to re-emerge in 2009 with an increase of 4.0 percentage points to $12.8 \%$ for any first-line drug resistance and an increase of 4.9 percentage points to $10.4 \%$ isoniazid resistance. Thus, the AAPC for rate of resistance to isoniazid increased by $13.3 \%$ from 2000 to 2009 (p<0.001, total: 365 cases). The rates and trends in rifampicin resistance and multidrug resistance increased similarly to those for isoniazid.

\section{Treatment outcome and mortality}

Treatment outcome was reported by 23 countries. The overall treatment success for the year cohorts 2002 to 2008 for which outcome data were available during the study period, was $80.5 \%$ for all culture-confirmed pulmonary paediatric TB cases $(n=2,958)$. For the seven high-incidence countries $(n=1,324)$, the overall treatment success was $87.3 \%$ with an increase from $81.5 \%$ in 2002 to $89.4 \%$ in 2008 and an AAPC of $1.6 \%$. For the 16 low-incidence countries $(n=1,634)$, the overall treatment success rate was $75.0 \%$. It should be noted, however, that the proportion of cases that were lost to follow-up was high in 2002 to 2008 , with as many as $27.6 \%$ in 2008 (Figure 8).

During 2000-2009, $1.6 \%$ of all culture-confirmed pulmonary paediatric TB cases died: a total of 160 children. High-incidence countries accounted for $76.9 \%$ of these deaths.

\section{Discussion}

Overall, in the EU/EEA, childhood TB is declining, as is TB in other age groups (see Figure 3). No significant differences in the childhood TB trends compared to the other age groups were observed.

However, the picture changes when looking separately at high-incidence and low-incidence countries (see Figure 4).

In high-incidence countries, over a 10 year period, there has been a decline and/or stabilisation of notifications in all paediatric age groups, with a particularly marked decrease in infants, suggesting a decline in recent transmission. In contrast, these rates have increased in low-incidence countries, but with a large variation over time, in particular for infants.

Separate correlation analyses for high-incidence and low-incidence countries to their five-year AAPC clearly demonstrated that childhood TB trends correlate better to the overall trends in high-incidence countries, compared with low-incidence countries. This could 
reflect two different epidemiological scenarios. The poor correlation between childhood and overall rates in low-incidence countries could be attributable to a number of factors. Childhood TB might be particularly sensitive to TB outbreaks in contrast to the overall epidemic trends. Since outbreaks, i.e. isolated events in a non-endemic setting, will not necessarily nor entirely reflect overall disease trends within a country, it is possible that paediatric TB increases due to outbreaks among children might occur against a background of low incidence and stable trends.

In high-incidence countries, where the epidemiological situation of TB is more uniform among the population, paediatric trends will reflect more directly overall TB transmission. In contrast, in low-incidence countries, the impact of foreign-born cases could affect this relationship, particularly if many of the cases are infected before entering the country and are thus unrelated to the local epidemiological situation.

The overall percentage of foreign-born childhood TB cases in the EU/EEA remains low (15.9\%). However, in low-incidence countries, it is similar (29\%) to that in the United States, where 31\% of cases between 1994 and $\mathbf{2 0 0 7}$ were attributable to foreign-born children [4]. Nevertheless, this percentage of TB cases among foreign-born children in low-incidence countries has remained stable, whilst the only marked increase in the paediatric population was recorded among nationalborn children under the age of five years (AAPC $4.4 \%$ ). A similar observation has been reported previously by a number of EU Member States [7,11], and could be a reflection of children being classified as national-born although born to foreign-born parents, and/or living in a foreign-born household, and thus exposed to a higher transmission risk.

Analysis of bacteriological confirmation of childhood TB cases emphasised that appropriate diagnosis remains a major challenge of childhood TB, even within the EU/ EEA. Although microbiologically confirmed cases in this area were frequent when placed in an international context $[12,13]$, the actual percentage of $16.9 \%$ signals an urgent need for improvement in microbiological diagnosis of TB in children. This becomes particularly important in view of the threat posed by multi- and extensively drug-resistant TB, and the need to rapidly initiate an appropriate treatment regimen.

Treatment outcome analysis for new pulmonary cultureconfirmed childhood TB cases was in line with that reported for the general population $[7,14]$. However, a better outcome performance in high-incidence countries was also evident. Whether this is attributable to better case management or better reporting, cannot be deduced from the available data.

Our data and analysis have a number of limitations which need to be taken into consideration when interpretating of our results. Despite its comprehensiveness,
TB surveillance in the EU/EEA relies on the quality of the surveillance systems in the Member States. The completeness and quality of external data cannot be measured directly at EU level (TESSy). In particular, the childhood TB data could be biased by over-diagnosis that is not uncommon in certain settings. At present we do not have appropriate data to evaluate quality of diagnostic practices and or sensitivities (commonly known as case detection), and if this differs between high- and low-incidence countries or where it has been constant over time. The variation in quality and case detection of TB surveillance data is assumed on the basis of published peer-reviewed articles on capturerecapture analysis reporting under notification $[15,16]$. This could in turn affect an interpretation of the real disease burden. With respect to the analysis of foreign-born versus national-born childhood TB burden, it would have been appropriate to carry out the analysis on disaggregated data, however the lack of reliable population denominators for the foreign-born populations in the EU/EEA made this impossible.

\section{Conclusions}

This work represents the first comprehensive attempt at an in-depth analysis of childhood TB epidemiology for the EU/EEA Member States. The data were deliberately presented in an aggregated fashion: it would have been impractical to comment on trends in single Member States because a number of these report very low case loads, affecting the significance of results.

Our findings agree with the international literature on the percentages of paediatric cases and notification rates (with rates peaking during infancy) $[4,17]$. The overall declining trend also mirrors what is observed in other similar settings [17]. However, if we focus on the low-incidence EU/EEA Member States, the trends are not consistently declining and contradict what is seen in other similar settings, such as the United States, where a constant decline has been recorded since 1994 in both foreign-born and native populations. [4]

The findings make it plausible to assume that within a context of overall epidemiological assessment, childhood TB trends can be used as a monitoring component. The recently launched EU TB monitoring framework proposes the trend in the ratio of TB in children versus adults as an EU/EEA epidemiological indicator [5]. The incidence of TB in children, especially the younger cohorts (under one year of age), is an indirect measure of the level of transmission in the community. Because young children have a much higher rate of primary disease progression, a lower transmission rate should be reflected in a decrease in the ratio of notification rates in children over adults. These features make childhood TB epidemiology worth exploring for the purpose of epidemiological monitoring.

The concept that childhood TB cases represent the results of recent transmission and infection is important from an advocacy point of view. Some authors 
have highlighted that complete TB elimination in the low-incidence European setting in not feasible because in our globalised times, importation of both active and latent cases might hamper local elimination. Even if this were true, elimination of transmission, particularly to children, within the borders of the EU should remain an achievable commitment.

Last but not least, it should be emphasised that surveillance data point towards suboptimal diagnostic practices in childhood TB in the EU/EEA. The low proportion of bacteriological confirmation of cases seriously limits childhood TB control and appropriate clinical management of cases. This, along with the need of interrupting transmission of TB from adults to children, needs to be addressed in order to progress towards elimination and to ensure the highest standards of TB care and control in a highly vulnerable population.

\section{References}

1. Nelson LJ, Wells CD, Global epidemiology of childhood tuberculosis. Int J Tuberc Lung Dis. 2004;8(5):636-47.

2. del Rosal T, Baquero-Artigao F, García-Miguel MJ, MéndezEchevarría A, López G, Aracil FJ, et al. Impact of immigration on pulmonary tuberculosis in Spanish children: a three-decade review. Pediatr Infect Dis J. 2010;29(7):648-51.

3. Kampmann B. Impact of immigration on pulmonary tuberculosis in spanish children. Pediatr Infect Dis J. 2010;29(7):652.

4. Menzies HJ, Winston CA, Holtz TH, Cain KP, Mac Kenzie WR. Epidemiology of tuberculosis among US- and foreign-born children and adolescents in the United States, 1994-2007. Am J Public Health. 2010;100(9):1724-9.

5. European Centre for Disease Prevention and Control (ECDC). Progressing towards TB elimination. A follow-up to the Framework Action Plan to Fight Tuberculosis in the EU. Stockholm: ECDC; 2010. Available from: http://www. ecdc.europa.eu/en/publications/Publications/101111_SPR Progressing_towards_TB_elimination.pdf

6. Walls T, Shingadia D. The epidemiology of tuberculosis in Europe. Arch Dis Child. 2007;92(8):726-9.

7. European Centre for Disease Prevention and Control/WHO Regional Office for Europe. Tuberculosis surveillance in Europe 2008. Stockholm: ECDC; 2010. Available from: http://www. euro.who.int/ data/assets/pdf file/0007/78856/E93600.pdf

8. Hollo V, Amato-Gauci A, Ködmön C, Manissero D. Tuberculosis in the EU and EEA/EFTA countries: what is the latest data telling us? Euro Surveill, 2009. 14(11):pii=19151. Available from: http://www.eurosurveillance.org/ViewArticle. aspx?Articleld $=19151$

9. European Centre for Disease Prevention and Control/WHO Regional Office for Europe. Tuberculosis surveillance in Europe 2009. Stockholm: ECDC; 2011. Available from: http://ecdc. europa.eu/en/publications/Publications/1103_TB_SUR_2009. pdf

10. Broekmans JF, Migliori GB, Rieder HL, Lees J, Ruutu P, Loddenkemper R, et al. European framework for tuberculosis control and elimination in countries with a low incidence. Recommendations of the World Health Organization (WHO), International Union Against Tuberculosis and Lung Disease (IUATLD) and Royal Netherlands Tuberculosis Association (KNCV) Working Group. Eur Respir J. 2002;19(4):765-75.

11. Abubakar I, Laundy MT, French CE, Shingadia D. Epidemiology and treatment outcome of childhood tuberculosis in England and Wales: 1999-2006. Arch Dis Child. 2008;93(12):1017-21.

12. Cruz AT, Starke JR. Clinical manifestations of tuberculosis in children. Paediatr Respir Rev. 2007;8(2):107-17.

13. Eamranond P, Jaramillo E. Tuberculosis in children: reassessing the need for improved diagnosis in global control strategies. Int I Tuberc Lung Dis. 2001;5(7):594-603.
14. Manissero D, Hollo V, Huitric E, Ködmön C, Amato-Gauci A. Analysis of tuberculosis treatment outcomes in the European Union and European Economic Area: efforts needed towards optimal case management and control. Euro Surveill, 2010. 15(11): pii=19514. Available from: http://www.eurosurveillance. org/ViewArticle.aspx?Articleld=19514

15. Cojocaru C, van Hest NA, Mihaescu T, Davies PD. Completeness of notification of adult tuberculosis in Iasi County, Romania: a capture-recapture analysis. Int J Tuberc Lung Dis. 2009;13(9):1094-9.

16. Pillaye J, Clarke A. An evaluation of completeness of tuberculosis notification in the United Kingdom. BMC Public Health. 2003;3:31.

17. Marais BJ, Gie RP, Schaaf HS, Hesseling AC, Obihara CC, Starke JJ, et al. The natural history of childhood intra-thoracic tuberculosis: a critical review of literature from the prechemotherapy era. Int J Tuberc Lung Dis. 2004;8(4):392-402. 